\title{
Wahrnehmung und Evaluierung des Balkan als Pulverfass im deutschsprachigen Pressediskurs
}

\section{Perception and Evaluation of the Balkans as a Powder Keg in the German-language Press Discourse}

\author{
Vjosa HAMITI' ${ }^{1}$, Milote SADIKU² (])
}

${ }^{1}$ Prof. Ass. Dr., University of Prishtina "Hasan Prishtina", Faculty of Philology, Department of German Language and Literature, Prishtina, Kosovo

${ }^{2}$ Prof. Asoc. Dr., University of Prishtina

"Hasan Prishtina", Faculty of Philology,

Department of German Language and

Literature, Prishtina, Kosovo

ORCID: V.H. 0000-0003-2916-516X; M.S. 0000-0001-5910-2974

\section{Corresponding author:}

Vjosa HAMITI,

University of Prishtina "Hasan Prishtina", Faculty of Philology, Department of German Language and Literature,

Prishtina, Kosovo

E-mail: vjosa.hamiti@uni-pr.edu

Submitted: 24.12 .2019

Revision Requested: 18.03.2020

Last Revision Received: 01.04 .2020

Accepted: 08.04.2020

Citation: Hamiti, V., Sadiku, M. (2020) Wahrnehmung und Evaluierung des Balkan als Pulverfass im deutschsprachigen Pressediskurs. Alman Dili ve Edebiyat Dergisi-Studien zur deutschen Sprache und Literatur, 43, 39-67.

https://doi.org/10.26650/sds|2019-0020

\begin{abstract}
DEUTSCH)
Im vergangenen Jahrhundert wird der Balkan im Allgemeinen und insbesondere ExJugoslawien in Medienberichten mit der Metapher "Balkan Pulverfass" beschrieben, als eine Gegend, in der es immer wieder zu Unruhen kam und weiterhin kommen könnte. Im alltäglichen und massenmedialen Diskurs hat die Verwendung von Metaphern eine Evaluierungsfunktion und bei der Darstellung von politisch relevanten Vorgängen eine persuasive Funktion, durch die Einstellungen und Bewertungen des Textproduzenten den Lesern vermittelt werden. In diesem Beitrag werden wir am Beispiel der Metapher „Pulverfass Balkan“ aufzeigen, wie die Metapher seit dem Ersten Weltkrieg zum Bewertungsmuster der deutschsprachigen Balkanwahrnehmung geworden ist. Im Mittelpunkt des Beitrages steht die Analyse der Metapher in den deutschsprachigen Berichterstattungen aus Zeitungen und Zeitschriften, die den Zeitraum vom Beginn des Ersten Weltkrieges bis zu Gegenwart umfassen. Ziel des Beitrages ist es zu zeigen, welche Funktionen die Metapher als Deutungsmuster im deutschsprachigen Mediendiskurs zur Sicherheitslage auf dem Balkan, genauer in ExJugoslawien übernommen hat. In diesem Beitrag wird zunächst der Begriff Metapher nach dem Ansatz der konzeptuellen Metapherntheorie und der Blending Theorie erläutert. Anschließend wird mit Schwarz-Friesel (2015) die metaphorische Äußerung „Pulverfass Balkan“ im Rahmen der kritischen Kognitionslinguistik (KKL) untersucht. Die Datengrundlage der Analyse bildet das Korpus aus online verfügbaren Archiven und Beiträgen gesammelten Kommentaren, Reportagen, Berichterstattungen und Interviews. Die Ergebnisse der Untersuchung zeigen, dass die metaphorische Äußerung Pulverfass Balkan ein viel breiteres Bedeutungspotential hat.
\end{abstract}

Schlüsselwörter: Metapher, Balkan, Pulverfass, Ex-Jugoslawien, Kriege, Pressediskurs

\section{ABSTRACT (ENGLISH)}

Media reports have employed the metaphor Pulverfass "Pulverfass Balkan (Balkan Powder Keg)" in the past century to describe the Balkans in general, and the former Yugoslavia in particular, as an area in which unrest has repeatedly occurred and might continue to occur. In everyday and mass media discourse, the use of metaphors has an evaluation function, whereas in the representation of politically relevant processes a persuasive function through which the attitudes and evaluations of the 
text producer are conveyed to the readers. In this article we will use the example of the metaphor "Pulverfass Balkan (Balkan Powder Keg)" to show how it has become a rating pattern for German-speaking Balkan perceptions since the First World War. The focus of this paper is the analysis of this metaphor in the German-language media reports from the beginning of the First World War to the present - a century later - with the aim of finding out which functions they serve as a pattern of interpretation in the German-language media discourse on the security situation in the Balkans, more specifically in the former Yugoslavia. The concept of the metaphor used in this article draws on both conceptual metaphor theory and blending theory. Schwarz-Friesel (2015) examines the metaphorical utterance of the Balkan powder keg in the context of critical cognitive linguistics (CCL). The corpus for this paper consists of archives and articles available online (such as comments, reports, and interviews). The potential significance of the metaphorical expression of the Balkan powder keg is examined on the basis of the selected corpus.

Keywords: Metaphor, Balkan, Powder Keg, Former Yugoslavia, Wars, Media Discourse

\section{EXTENDED ABSTRACT}

In this article, the analysis of the metaphor "Pulverfass Balkan (Balkan Powder Keg)" shows how this metaphor has become an evaluation pattern vis-à-vis the Balkans amongst German-speakers since the First World War. The focus of the article is the investigation of the usage of this metaphor in German-language news accounts covering the Balkans from the beginning of the First World War to the present, more than a century later. The aim of the investigation is to find out which functions this metaphor has taken on as a pattern of evaluation/interpretation in German-language media discourse on the security situation in the Balkans, more specifically in the former Yugoslavia. At the same time, the intensity of use of this metaphor in times of acute crises shall be examined in comparison to times of low intensity conflict. This study also aims to show how the perception of the Balkans has developed in German-speaking countries. Routinely resorting to this metaphor, media reports have described the Balkans in general, and the former Yugoslavia in particular, as an area in which unrest has repeatedly occurred and might continue to occur. Using the metaphors of powder keg, fuse and spark, the recipient is fed with violence, threat and even fear scenarios that can trigger emotional concepts such as anxiety and fear.

This article assumes that the metaphor "Pulverfass Balkan (Balkan Powder Keg)" describes a territory in which there is a high voltage that could lead to an explosion at any time. The causes of events that lead to the explosion of the powder keg are established discursively by the semantics of the lexical units "throw/be a spark" and "put a fuse", as in the idioms "den Funken ins Pulverfass werfen/schleudern ('throw a spark into the powder keg'); der Funke im Pulverfass sei ('the spark in the powder keg') and die Lunte ans Pulverfass legen ('put the fuse on the powder keg')". 
On the basis of evidence from a press coverage corpus (109 documents), this paper examines how the metaphorical expression "powder keg" has become the evaluation/ interpretation pattern of the Balkans and to what extent this interpretation pattern can lead to an intensification of the threat potential. The corpus consists of media coverage in times of acute crises (World War I and post-Yugoslav wars) and in times of lack of them. In this way we want to determine whether the metaphorical expression "powder keg" in German-language media discourse has the same vitality and the same potential in wartime as in times of low conflict. The outbreak of WWI is considered a discursive event, expressed widely by the "powder keg" metaphor.

The classic understanding of the metaphor is of a linguistic phenomenon that serves to embellish speech in a poetic and rhetorical way. Since ancient times, it has been the subject of investigation not only in linguistic but in many other scholarly fields. Metaphors are no longer regarded as mere ornaments of language in the scholarship on the metaphor. Especially since the groundbreaking publication Metaphors we live by (1980) by Lakoff and Johnson, which has been around for almost 40 years, the discussion of metaphors has been booming in many specialist disciplines. As the main representative of conceptual metaphor theory, which originated in the theoretical framework of cognitive linguistics, Lakoff and Johnson (2008 [1997]) relate the concept of metaphor to the conceptual level. This approach is based on the assumption that metaphors structure our everyday life and have a great impact on the way we think and act, i.e. language and thinking are subject to the same cognitive principles. In cognitive metaphor theory, complex and abstract phenomena can be summarized in familiar and elementary understandable concepts: conceptual metaphors actively participate in the processing of experiences.

Press documents from ANNO - Historical Austrian Newspaper and Magazine Archives - serve as the material basis for the investigation for the period of the First World War. The reports from 1945 to the present consist of documents from the online archives of Der Spiegel, Tages-Anzeiger, St. Galler Tagblatt, Die Zeit, Die Presse and Euronews.

The analysis shows that the metaphor "Pulverfass Balkan (Balkan Powder Keg)" inferred the following conceptual features: "instability", "primitiveness", "unreliability", "ethnic and religious mixture", "violence", "corruption", "lack of democracy" and "fundamentalist Islamism". The use of the metaphor "Pulverfass Balkan (Balkan Powder $\mathrm{Keg})^{\prime \prime}$ in media discourse has the function to convey attitudes and evaluations of the West as well as to convey opinions about the situation in the Balkans in general. 
In summary, it can be said that the Balkans have been referred to as a "powder keg" for more than a century due to political and historical events. In the documents examined, the metaphorical expression "powder keg" has always been used for the whole Balkans, the instability of which threatens the whole of Europe. 


\section{Einleitung}

In dem vorliegenden Beitrag wird anhand der Analyse der Metapher „Pulverfass Balkan“ versucht aufzuzeigen, wie diese Metapher seit dem Ersten Weltkrieg zum Bewertungsmuster der deutschsprachigen Balkanwahrnehmung geworden ist. Im Mittelpunkt des Beitrags steht die Untersuchung dieser Metapher in der deutschsprachigen Berichterstattung vom Beginn des Ersten Weltkrieges bis zur Gegenwart, also mehr als ein Jahrhundert danach. Ziel der Untersuchung ist herauszufinden, welche Funktionen diese Metapher als Deutungsmuster im deutschsprachigen Mediendiskurs zur Sicherheitslage auf dem Balkan, genauer in ExJugoslawien, hat. Außerdem soll durch diese Untersuchung aufgezeigt werden, wie sich die Wahrnehmung des Balkans im deutschsprachigen Raum entwickelt hat. Im vergangenen Jahrhundert wird der Balkan im Allgemeinen und insbesondere ExJugoslawien in Medienberichten mit dieser Metapher beschrieben, als eine Gegend, in der es immer wieder zu Unruhen kam und weiterhin kommen könnte. Mittels der Verwendung der metaphorischen Äußerungen „Pulverfass, Lunte und Funke" werden dem Rezipienten Gewalt-, Bedrohungs- und sogar Angstszenarien vermittelt, die Emotionskonzepte wie Angst und Furcht auslösen können?.

Die vorliegende Untersuchung hat ergeben, dass die Verwendung dieser Metapher im westlichen Raum schon die Zeit vor dem Ersten Weltkrieg datiert, jedoch ist sie mit dem Kriegsausbruch stärker im westlichen Vokabular und Bewusstsein vertreten. Dadurch wird die Metapher „Pulverfass Balkan“ zu einem Bewertungsmuster der Balkanwahrnehmung im westlichen Raum. Diese Metapher beruht auf folgende Faktoren: Instabilität, Primitivität, Unzuverlässigkeit, ethnische und religiöse Durchmischung, Gewalt, Korruption und mangelnde Demokratie. Mit Czachur (2015) können wir festhalten, dass auf Grund dieser Faktoren die Metapher „Pulverfass Balkan“ als Metapher ,der langen Dauer' bzw. Makro-Metapher ${ }^{2}$ zu bezeichnen ist, „[...] weil sie jederzeit als Bewertungsmuster, als Argumentationstopos, als Bild aktiviert werden kann, d.h. ihr semantisches Potenzial sowie ihre sozial anerkannte, stillschweigende Wirkungsmacht sind stets abrufbar" (Czachur, 2015, S. 233).

1 Mehr über Emotionskonzepte Angst und Furcht siehe auch Wierzbicka (1999), Ulich \& Mayring (2003), Schwarz-Friesel (2013 [2007]).

2 Kövecses (2010 [2002], S. 57) hat die Diskussion über die "megametaphors" ("extending metaphors") and "micrometaphors" in literatischen Texten eingeführt, worauf das Verständnis der Bewertungsmetapher als Makro-Metapher basiert. 
Metaphern sind sprachliche Äußerungen, bei denen eine Relation zwischen zwei unterschiedlichen Konzepten ausgedrückt wird. So wird der Balkanraum als ein Behälter von Sprengstoff (Pulverfass) referenzialisiert, indem Kenntnisse aktiviert werden und bestimmte mentale Modelle entstehen. Durch die Semantik von „Pulverfass" werden konzeptuelle Merkmale wie „(Hoch-) Spannung, Instabilität, Explosion, Konflikt” inferiert.

Der Balkan wurde in unzähligen Arbeiten aus historischer, sozialer und politischer Perspektive untersucht ${ }^{3}$. In den meisten Arbeiten wird der Balkan mit der Metapher „Pulverfass" bewertet (s. Kap. 2.3). Jedoch sind wir auf keine Studie gestoßen, die die Metapher „Pulverfass Balkan“ aus der linguistischen Perspektive untersucht. Die vorliegende Untersuchung will diese Forschungslücke schließen, indem wir das Vorkommen der Metapher „Pulverfass Balkan“ in den deutschsprachigen Medien linguistisch untersuchen.

\section{Theoretisch-methodische Grundlagen}

\subsection{Methodische Herangehensweise}

In diesem Beitrag wird davon ausgegangen, dass die Metapher „Pulverfass Balkan“ ein Territorium beschreibt, in dem eine Hochspannung herrscht, die jederzeit zur Explosion führen könnte. Das Hervorrufen von Ereignissen, die zur Explosion des Pulverfasses führen, wird diskursiv durch die Semantik der lexikalischen Einheiten „Funke werfen/sein und Lunte legen“ etabliert, wie in den Redewendungen "den Funken ins Pulverfass werfen/schleudern; der Funke im Pulverfass sein ${ }^{4}$ und die Lunte ans Pulverfass legen ${ }^{5 \prime .}$

In diesem Beitrag soll anhand von Belegen aus einem Pressetext-Korpus (109 Belege) erörtert werden, wie der metaphorische Ausdruck "Pulverfass" zum Bewertungsmuster des Balkans geworden ist und inwiefern dieses Deutungsmuster und Bewertungskriterium zu einer Intensivierung des Bedrohungspotenzials führen kann. Den Ausgangspunkt für die Zusammenstellung des Korpus bilden einerseits die Zeiten akuter Krisen (Erster

3 Siehe u.a. Veichtlbauer (2001), Todorova (2009), Sundhaussen (1999), Mišev (2001), Keyserling (1931).

4 „Den Funken ins Pulverfass werfen“ ,durch etw. Unbedachtes, Geringfügiges ein Verhältnis, eine Lage so weit verschlimmern, dass es zum offenen Konflikt kommt' (Duden Universalwörterbuch, 2015 [1983], S. 656); „,den Funken ins Pulverfass schleudern 'einen schwelenden Konflikt zu einem offenen Streit werden lassen'; der Funke im Pulverfass sein 'das auslösende Moment für einen Streit, Konflikt sein" (Duden 11, 2013 [1992], S.242).

5 „Die Lunte ans Pulverfass legen“, durch eine bestimmte Äußerung, Handlung einen schwelenden Konflikt zum offenen Streit werden lassen' (Duden 11, 2013 [1992], S. 484). 
Weltkrieg und postjugoslawische Kriege), andererseits die konfliktarmen Zeiten im ehemaligen Jugoslawien (Zeitraum nach der Millenniumswende bis zur Gegenwart). Durch die Einteilung des Korpus in diese zwei Bereiche wollen wir ermitteln, ob der metaphorische Ausdruck „Pulverfass“ auch in konfliktarmen Zeiten im deutschsprachigen Mediendiskurs die gleiche Vitalität und das gleiche Wirkungspotenzial wie zu Kriegszeiten aufweist. Der Ausbruch des Ersten Weltkriegs gilt als diskursives Ereignis, das die in der vielgebrauchten Metapher vom „Pulverfass” geprägt hat. Zurecht betont Geulen (2002, S. 77), dass den metaphorischen Ausdruck "Pulverfass" "kaum ein anderer Krieg so herbeigeredet" hatte, da er bereits zum damaligen Vorkriegsdiskurs gehörte.

Als Materialgrundlage der Untersuchung für den Zeitraum des Ersten Weltkrieges dienen Pressebelege aus ANNO - Historische österreichische Zeitungen- und Zeitschriften-Archive ${ }^{6}$. Die Berichterstattungen ab 1945 bis zur Gegenwart besteht aus Belegen aus den Online-Archiven von Der Spiegel, Tages-Anzeiger, St. Galler Tagblatt, Die Zeit, Die Presse und Euronews ${ }^{7}$.

Mit Schwarz-Friesel (2015) wird in diesem Beitrag die metaphorische Äußerung „Pulverfass Balkan“ im Rahmen der kritischen Kognitionslinguistik (KKL) untersucht:

Gemäß der prozeduralen Perspektive der KKL sind sprachliche Äußerungen als Spuren der mentalen Aktivität, die Aufschluss über Kenntnisse, Einstellungen und Beurteilungen der Sprachbenutzer geben (können), zu betrachten (Sprachproduktionsperspektive). (Schwarz-Friesel, 2015, S. 147)

Unsere Analyse des Bedeutungspotenzials der metaphorischen Äußerung „Pulverfass Balkan“ bzw. „Balkan Pulverfass Europas” beginnt mit der Erfassung ihrer öffentlichen Thematisierung im Mediendiskurs, denn Metaphern spielen eine besondere Rolle in der massenmedialen Darstellung von Politik. Die durch den metaphorischen Ausdruck "Pulverfass" evozierten mentalen Modelle können im Rahmen der Presseberichterstattung eine große Wirkung auf kollektive Meinungsbildungsprozesse haben. Durch ihre Verwendung werden komplexe gesellschaftliche Themen vereinfacht und auf eindrückliche Weise veranschaulicht (Skirl \& Schwarz-Friesel, 2013, S. 72).

6 ANNO ist der virtuelle Zeitungslesesaal der Österreichischen Nationalbibliothek. Hier kann in historischen österreichischen Zeitungen und Zeitschriften online geblättert und gelesen werden. Sie datieren ab 1894 bis 2000. [http://www.anno.onb.ac.at/ (26.03.2020)] Als Suchbegriffe wurden „Pulverfass u. Balkan“ verwendet.

7 Auswahlkriterien für die Erstellung des Korpus für diesen Zeitraum waren folgende Suchbegriffe: „Balkan, Pulverfass, Jugoslawien, Kosovo, Serbien, Bosnien, Mazedonien“, in den folgenden online Medien: www.spiegel. de; www.tagesanzeiger.ch, www.zeitonline.de, www.diepresse.com, www.euronews.com, www.tagblatt.ch. 


\subsection{Begriffsbestimmung der Metapher}

Das klassische Metaphernverständnis betrachtet die Metapher als sprachliches Phänomen, das der poetisch-rhetorischen Ausschmückung der Rede dient (vgl. Skirl \& Schwarz-Friesel, 2013, S. 3; Becker, 2014, S. 199). Sie ist seit der Antike Untersuchungsgegenstand nicht nur linguistischer, sondern vieler anderer wissenschaftlicher Disziplinen. Metaphern werden in der Metaphernforschung nicht mehr als bloße Ornamente der Sprache betrachtet. Besonders seit der fast 40 Jahre zurückliegenden bahnbrechenden Publikation Metaphors we live by (1980) von Lakoff und Johnson erfährt die Auseinandersetzung mit Metaphern in vielen Fachdisziplinen einen Aufschwung (vgl. Spieß \& Köpcke, 2015, S. 1). Der Ansatz von Lakoff und Johnson wird als eine neue Stoßrichtung innerhalb der Metaphernforschung bezeichnet und „bildet gleichsam einen wesentlichen Grundpfeiler für die Entstehung der Kognitiven Linguistik"(Schröder, 2012, S. 26). Als Hauptvertreter der Konzeptuellen Metapherntheorie, die im theoretischen Rahmen der Kognitiven Linguistik entstand, beziehen Lakoff und Johnson (2008 [1997]) den Metaphernbegriff auf die konzeptuelle Ebene. Dieser Ansatz basiert auf der Annahme, dass Metaphern unser Alltagsleben strukturieren und einen großen Einfluss auf die Art und Weise haben, wie wir denken und handeln, d.h. Sprache und Denken unterliegen den gleichen kognitiven Prinzipien. In der kognitiven Metapherntheorie sind komplexe und abstrakte Phänomene in vertraute und elementar verständliche Konzepte zu fassen: konzeptuelle Metaphern nehmen aktiv an der Verarbeitung von Erfahrungen teil. Metaphern kristallisieren „allgemeine Vorstellungen, Werte und Erwartungen und übertragen sie in Bilder, die ein emotives und persuasives Potenzial haben und komplexe Konzepte verständlicher, anschaulicher machen" (Ghilarducci, 2017, S. 183). Nach Ziem (2015) sind konzeptuelle Metaphern konventionalisierte und lexikalisierte Einheiten, die sich dadurch auszeichnen, , dass sie in unserem kognitiv-konzeptuellen System so fest verankert sind, dass ihr metaphorischer Gehalt leicht übersehen werden kann." (Ziem, 2015, S. 53).

Nach Schröder (2012, S. 63) hat die Konzeptuelle Metapherntheorie eine tiefe und Impuls-gebende Wirkung auf die Human-, Sozial- und sogar Naturwissenschaften ausgeübt. Der Ansatz der Konzeptuellen Metapherntheorie wurde in der sprachwissenschaftlichen Forschung durch verschiedene Publikationen vielfach erprobt, diskutiert, ausgearbeitet und erweitert. Obwohl der konzeptuelle Ansatz in der Linguistik bis heute als der wichtigste Beschreibungsrahmen für Metaphern gilt, ist er dennoch vielfach kritischen Einwänden ausgesetzt gewesen (vgl. Schröder, 2012, S. 63-78). So z.B. 
wird dieser Ansatz bei Schwarz-Friesel wegen erheblichen (theoretischen und methodischen) Schwächen immer wieder kritisiert (vgl. dazu Schwarz-Friesel, 2015, S. 145-146; 2014, S. 57). Sie betrachtet Metaphern nicht als konzeptuelle sondern als sprachliche Konstruktionen ${ }^{8}$.

Die Grundthese der konzeptuellen Metapherntheorie stellt Metaphern als ein kognitives Phänomen dar, in dem eine Domäne den Zielbereich (target domain) und die andere den Herkunftsbereich (source domain) der metaphorischen Projektion bildet. Diese "Unidirektionalitätsthese" vernachlässigt die Frage, "was passiert, sobald zwei Domänen in einem bestimmten Kommunikationskontext und im aktuellen Sprachgebrauch gekoppelt werden" (Schröder, 2012, S. 66), denn es kommt im Gebrauchskontext eigentlich zu einer Interaktion von Zielbereich und Herkunftsbereich. Von der unidirektionalen konzeptuellen Metapherntheorie grenzt sich die Theorie der konzeptuellen Interaktion, die sogenannte Blendingtheorie, ab, "da sie eine Vielzahl vergleichbarer metaphorischer wie nicht-metaphorischer Phänomene beschreiben kann“ (Liebert, 2014, S. 27). Diese Metapherntheorie wird besonders in den Fällen bevorzugt, in denen es schwierig ist zu entscheiden, ob eine Metapher vorliegt oder nicht. Die Bildung der Blendingtheorie geht auf Fauconnier (1997) bzw. Fauconnier \& Turner (2002) zurück, und nimmt in der deutschsprachigen Sprachwissenschaft erst allmählich ihren Platz ein (vgl. Liebert, 2015, S. 113). Die Blendingtheorie hat die sourceto-target-Richtung nicht angenommen, sie stellt einen Spezialfall der nicht-gerichteten Relation dar, wo mehr als zwei Konzepte an der Bedeutungskonstruktion mitwirken. Die Projektionsrichtung nach dem Blendingkonzept ist bidirektional. Fauconnier (1997) entwirft ein vierteiliges Netzwerk-Modell, das aus zwei „inputs" (Herkunftsbereich und Zielbereich), einem "generic space" (in dem die Analogien der „inputs" motiviert werden) ${ }^{9}$ und dem „blended space” (als Resultat dieses Prozesses) besteht. Im Bereich „blended space gehen zwei „inputs" eine Interaktion ein, aus der eigene semantische Strukturen hervorgehen: „The blend has emergent structure not provided by the inputs" (Fauconnier, 1997, S. 150). Die Blendingtheorie macht die Konzeptuelle Metapherntheorie nicht ungültig, jedoch, nach Liebert (2015), überflüssig, denn mit der Blendingtheorie können Metaphern immer beschrieben werden ${ }^{10}$.

8 Ähnlich auch bei Skirl und Schwarz-Friesel (2013), wo der Metaphernbegriff nur im Hinblick auf die sprachliche Ebene untersucht wird und nicht im Hinblick auf die konzeptuelle Ebene.

9 Fauconnier (1997, S. 152): "The generic space contains what the inputs have in common.”

10 „Daher kann man aber auch durchaus noch - insbesondere um die Lesbarkeit zu erhöhen - von Metaphern in der CMT-Terminologie sprechen, wenn man weiß, dass Metaphern eine spezifische Form von Blendings darstellen und auch jederzeit mit Blendingkategorien beschrieben werden können." (Liebert, 2015, S. 113). 
Nach der Blendingtheorie können wir die Metapher „Pulverfass Balkan“ wie folgt analysieren. Mit dieser Metapher wird der "blended space Kriegsausbruch" und „Lebensbedrohung" ausgelöst, der in den beiden Inputs nicht vorkommt. Die Ähnlichkeiten, die vom Herkunftsbereich „Pulverfass“ und vom Zielbereich „Balkan“ motiviert sind, nämlich "Instabilität", „Hochspannung ", „Lunte“, ,Funke“, sind im Bereich „generic space" enthalten.

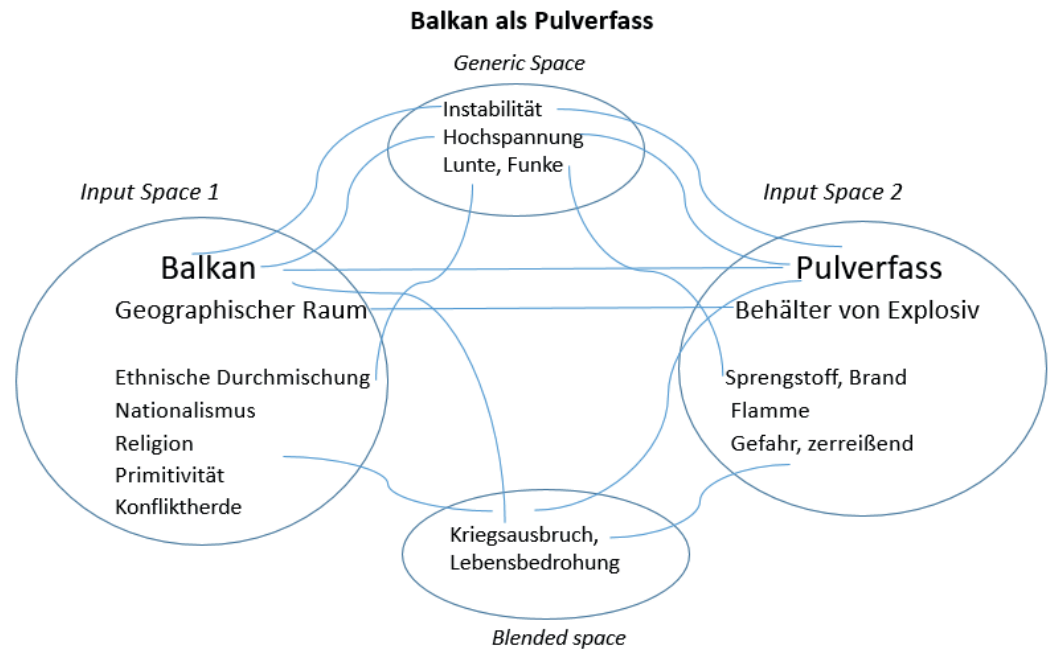

Fig 1. Blend von „Balkan als Pulverfass“ (nach Fauconnier \& Turner, 2002)

In der Linguistik besteht kein Konsens über den Metaphernbegriff, denn es scheint schwierig zu sein, eine Präzisierung dessen zu geben, was eine Metapher ist (vgl. Schmitt, 2017, S. 39). In der einschlägigen Literatur findet man unterschiedliche Metaphernbegriffe. Skirl und Schwarz-Friesel (2013, S. 5) zufolge weichen die Begriffe der Metapher in der Alltagssprache und in verschiedenen Wissenschaftsdisziplinen beträchtlich von der sprachwissenschaftlichen Bestimmung ab. Skirl und Schwarz-Friesel definieren Metapher als eine spezifische Form des nicht-wörtlichen Gebrauchs eines sprachlichen Ausdrucks in einer bestimmten Kommunikationssituation und grenzen den metaphorischen Sprachgebrauch von Vergleichen und von metonymischem Sprachgebrauch ab (vgl. Skirl \& Schwarz-Friesel, 2013, S. 11).

Schwarz-Friesel (2015) betrachtet die Metaphern als die wichtigsten sprachlichen Mittel, die das Verstehen von abstrakten und schwierigen Phänomenen und Gegenstandsbereiche (wie kognitive und soziale Prozesse, Emotionen, technische Erneuerungen, Ideologien etc.) erleichtern. Nach ihr drücken Metaphern nicht nur Konzeptualisierungen aus, sondern sie 
„konstituieren auch spezifische Konzeptualisierungen, die über die jeweilige Semantik der Äußerungen vermittelt werden" (Schwarz-Friesel, 2015, S. 143).

\subsection{Geschichte der Metapher „Pulverfass Balkan“}

Mit dem Begriff „Balkan“ (aus dem Türkischen: „Gebirge“) wird eine identifizierende geographische Beschreibung für einen Raum, der auf der Halbinsel südlich der Donau liegt, bezeichnet. Im Lexikon zur Geschichte Südosteuropas (Sundhaussen \& Clewing, 2016, S. 110) werden dem Balkan sechs Bezeichnungen zugeschrieben: 1) Balkan-Gebirge, 2) Balkan-Halbinsel, 3) Westbalkan, 4) Balkan als historischer Raum, 5) Balkan als Kulturlandschaft, 6) Balkan als „mental map“. In der Fachliteratur herrscht die Meinung, dass selbst der Begriff „Balkan“ und/oder "Balkanisierung"11 eine Metapher mit negativer Konnotation ist (vgl. hierzu Koschmal, 2009; Todorova, 2009; Bjelić, 2002). Im deutschen Sprachgebrauch stehen nach Koschmal (2009, S. 448) und Sundhaussen (1999, S. 626) die Begriffe „Balkan“, „Balkanisierung" usw. auch für „Anarchie“, "Zerstückelung größerer politischer und wirtschaftlicher Einheiten“, "Fragmentierung" und "Instabilität". Über die Menschen sowie über die herrschenden Zustände auf dem Balkan wurde mit Verachtung gesprochen. Also, alles, was mit dem Lexem „Balkan“ zusammenhängt, wurde stark negativ konnotiert. Ähnliche Beobachtungen hat auch Goldsworthy (2002, S. 34) gemacht, die schreibt, dass "[...] the Balkans have become nothing but a metaphor for conflict, incivility, and violence". Todorova $(2009$, S. 57) stellt fest, dass die eigenständige Rhetorik über den Balkan vom Westen erfunden und importiert wurde und das pejorative Balkan-Bild eine westliche Konstruktion ist, um sich vom „Andersartigen“ innerhalb Europas zu distanzieren ${ }^{12}$. Sie unterstreicht, dass der Balkan an der Kreuzung zwischen zwei unterschiedlichen Welten (dem Westen und dem Osten) liegt und unterschiedliche Kulturen, Sprachen, Traditionen und sogar Zivilisationen verbindet. Ihr zufolge evoziere die Bezeichnung „Balkan“ immer das Bild einer Brücke als eine Vergleichsmetapher zwischen Ost und West, zwischen Europa und Asien (Todorova, 2009, S. 16) ${ }^{13}$.

11 Nach Todorova (2009, S. 33) tauchte der Begriff „balkanization“ erstmals in der New York Times am 20. Dezember 1918 auf.

12 "As in the case of the Orient, the Balkans have served as a repository of negative characteristics against which a positive and self-congratulatory image of the "European" and the "West" has been constructed. With the reemergence of East and orientalism as independent semantic values, the Balkans are left in Europe's thrall, anticivilization, alter ego, the dark side within" (Todorova, 2009, S. 188).

13 Diese Balkan-Rhetorik entdeckt Todorova in den von ihr untersuchten Reiseberichten über den Balkan. Der Balkan wird dabei als eine „wavering" Form, eine Mischung aus Ost und West dargestellt, dessen Völker weder Orientalen noch Europäer sind, weil "they have not freed themselves from the vices of the East nor acquired any of the virtues of the West" (Todorova, 2009, S. 125). 
Der Balkan wird häufig als Synonym für unzivilisierte primitive Menschen (Bauern), ethnische und religiöse Durchmischung, patriarchale Gesellschaften und vieles mehr angesehen. Für Kaplan (2000) bedingen die religiöse und kulturelle Durchmischung auf dem Balkan das Reden vom „Pulverfass": ${ }^{14}$

In other words, the Balkans, a powder keg for nation-state war at the beginning of the twentieth century, could be a powder keg for cultural war at the turn of the twenty-first: between Orthodox Christianity (represented by the Serbs and a classic Byzantine configuration of Greeks, Russians, and Romanians) and the House of Islam (Kaplan, 2000, S. 29).

Keyserling (1931) hingegen fasst das Wesen des „Balkan“ und die Bezeichnung „Balkanisierung" wie folgt zusammen:

So, nicht anders liegt, von außen her betrachtet, das Balkan Problem. - Soweit ich sehe ist dessen sinnbildlicher Sinn von zwei Ansatzpunkten her am besten zu fassen: erstens der allgemein als wahr geltenden Behauptung, dass der Balkan das Pulverfass Europas sei. Zweitens von der Tatsache besonders elementarer und unausgleichbarer Völkerfeindschaft. Dass der Balkan das Pulverfass Europas ist, liegt offenbar an letzterem, nicht am ersteren. (Keyserling, 1931, S. 337)

Dieses negative Balkan-Bild gab es aber im Zeitalter der Romantik nicht, denn damals wurde der Balkan mit Bewunderung beschrieben. Das Balkan-Bild veränderte sich besonders seit dem ausgehenden 19. und dem beginnenden 20. Jahrhunderts, indem es „mit Primitivität und Gewaltbereitschaft" verbunden wurde (Sundhaussen, 1999, S. 627) ${ }^{15}$. Sundhaussen und Clewing (2016, S. 116) eruieren, dass der Balkan „als negative Alteritätsfolie des „Westens" v.a. in Zeiten akuter Krisen im Balkanraum fungiert", was während konfliktarmer Zeiten nicht der Fall sein sollte.

Das pejorative Balkan-Bild erlebte Anfang der 1990er Jahre eine Neuauflage. Als Auslöser werden die Darstellungen westlicher Massenmedien zum Krieg in Ex-

14 Im Duden Universalwörterbuch (2015 [1983], S. 1401) wird der Begriff „Pulverfass“ als ein,Fass für Schießpulver' definiert, und die Redewendung „, auf einem/dem Pulverfass sitzen“ bedeutet, ,sich in einer spannungsreichen, gefährlichen Lage befinden“. Dagegen wird im albanischen Online-Bedeutungswörterbuch der Begriff „Pulverfass" als „ein Ort/Land“ beschrieben, „wo der Krieg jeden Augenblick ausbrechen könnte” (http://www. fjalori.shkenca.org/. Zugriff: 06.03.2018). Ähnlich wie im Albanischen wird der Begriff „Pulverfass” auch im bulgarischen Bedeutungswörterbuch erklärt (vgl. hierzu Mišev, 2001, S. 224).

15 Ähnliche Wahrnehmungen des Balkans sind u.a. bei Todorova (2009); Koschmal (2009) zu finden. 
Jugoslawien betrachtet. Dadurch wurde die Diskussion über die Wahrnehmung des Balkans in westlichen Gesellschaften aktualisiert, in dem er "als besonders gewaltbereit und rückständig" (Sundhaussen \& Clewing, 2016, S. 116) galt ${ }^{16}$.

Es ist genau der politische und gesellschaftliche Kontext, in dem sich der Balkan seit dem 19. Jahrhundert befand, der die Entstehung der Metapher "Pulverfass Balkan“ förderte. Dem Balkan, dem „Pulverfass Europas“, sind die Merkmale Instabilität und Prozessualität eigen (vgl. Goldsworthy, 2002; Koschmal, 2009; Paris, 2002). Der Balkan wird zum Schauplatz verschiedenartiger Auseinandersetzungen zwischen Kulturen, Religionen und Völkern. In diesem Zusammenhang wird häufig auch der Anfang des

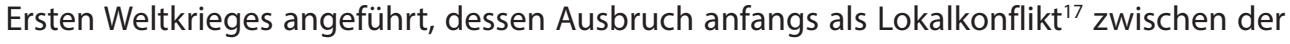
Donaumonarchie und Serbien angesehen wurde. Der Ausbruch des Ersten Weltkriegs wird von Historikern als Folge „eines ganzen Systems von Feindschaften"18 verstanden, das sich in der vielgebrauchten Metapher vom „Pulverfass" ausgeprägt hat. Allerdings herrscht auch die Meinung, dass der Balkan schon vor dem Ausbruch des Ersten Weltkrieges als „Pulverfass" galt (mehr dazu bei Goldsworthy, 2002, S. 27-28; Kaplan, 1993; Keisinger, 2008, S. 65; Geulen, 2002, S. 77).

Die lexikalische Einheit „Pulverfass” bezeichnet nach Koschmal (2009, S. 449) eine „unmittelbar bevorstehende, besonders heftige Veränderung“. Er hebt hervor, dass die

16 Der Historiker Holm Sundhaussen gibt diesbezüglich folgende Beschreibung: „Nicht alle negativen BalkanBilder sind falsch, nur weil sie negativ sind. [...] Rationale Kriterien sind logisch und berechenbar. Sie sind weder "schicksalhaft" noch "ganzheitlich", sondern diskutierbar und verhandelbar. Das Argumentationsmuster der jugoslawischen Konfliktparteien weisen eine andere Qualität auf: Sie sind überfrachtet mit Mythen und eschatologischen Alles-oder-Nichts-Positionen, die nur in toto angenommen oder abgelehnt werden können - Positionen, die nicht verhandelbar sind, denn über (vermeintliches) Sein oder Nichtsein, über Leben und Tod verhandelt man nicht." (Sundhaussen, 1999, S. 652).

17 Dennoch konnte der damalige Lokalkonflikt sich zum Weltkrieg entwickeln, nicht zuletzt wegen des Balkans an sich, sondern u.a. wegen der damals bestehenden Allianzen und "Schutzverträge" zwischen den mächtigen Nationen im Westen.

18 Sundhaussen und Clewing (2016) geben folgende Erläuterung: „Indem sich Ö.-U. mit dem Zweibundvertrag seit 1879 außenpol. stark an das Dt. Reich anlehnte, vermochte es die v. Russland ausgehenden Gefahren eines panslavistischen $(\rightarrow$ Panslawismus) $\rightarrow$ Irredentismus bis 1914 einzudämmen $u$. der Doppelmonarchie eine lange Friedenszeit zu sichern. Allerdings wurde diese v. einer im letzten Jahrzehnt vor 1914 zunehmenden Zahl v. Konflikten überschattet, die im Kern eine immer stärkere außenpol. Isolierung der beiden Mittelmächten Deutschland u. Ö.-U. anzeigten u. die für den Kriegsausbruch 1914 kennzeichnende Mächtekonstellation vorbereiteten. Der seit 1903 eskalierende Konflikt mit Serbien u. die $\rightarrow$ Annexionskrise v. 1908/09, in der die Wiener Diplomatie mit ihrer leichtfertig-oberflächlichen Einschätzung ihrer eigenen bescheidenen Möglichkeiten $u$. der weltpol. Zusammenhänge erstmals einen europaweiten Konflikt heraufbeschwor, hat Ansehen u. Autorität der Regierungen in Wien u. Budapest nach innen wie nach außen erheblich untergraben. Die erbitterte Feindschaft zw. Ö.-U. u. Serbien führte am 28. Juni 1914 zum Attentat des bosnischen Serben Gavrilo Princip in Sarajevo, bei dem der österr.-ung. Thronfolger Franz Ferdinand u. seine Gattin getötet wurden ( $\rightarrow$ Attentat v. Sarajevo)." (Sundhaussen \& Clewing, 2016, S. 685-686). 
Metapher "Pulverfass" Balkan einen Übergang, eine Prozessualität thematisiert und dass sie die Außenperspektive ${ }^{19}$ einschließt. Koschmal (2009) zählt diese Metapher zur Reihe der Europametaphern, doch mit radikalisierenden Merkmalen. Paris (2002) hingegen zählt die Metapher „Balkan powderkeg“ zu den Kriegsmetaphern ${ }^{20}$.

\section{Korpusanalyse}

\subsection{Analyse der Pressebelege akuter Krisen: Erster Weltkrieg und postjugoslawische Kriege}

Texte werden aufgrund von spezifischen Vorkenntnissen und Konzeptualisierungen produziert und rezipiert (vgl. Schwarz-Friesel, 2014, S. 53). Wie oben angedeutet, können sprachliche Äußerungen bei den Rezipienten spezifische kognitive Repräsentationen aktivieren und mentale Modelle entstehen lassen. So nimmt z.B. in (1) der Textproduzent die Opferperspektive ein, wenn er den Sachverhalt "das Attentat auf den Thronfolger" schildert und ihn als ein "abscheuliches, grausames Verbrechen gegen die Menschheit" negativ konzeptualisiert und entsprechend verbalisiert. Dahingegen wird in (2) die Konzeptualisierung und die Einstellung zum gleichen Sachverhalt mit einem Fabeltier, der "Hydra“21, versprachlicht. Durch die Referenzialisierung vom Konzept „Kriegsauslöser als einem Fabeltier“, als einem unbesiegbaren mythischen Ungeheuer, wird noch eine zusätzliche dämonische Dimension eröffnet. Durch die Semantik des Lexems "Hydra“ werden Komponenten wie "lebensbedrohlich", "schwer kontrollierbar" aktiviert (vgl. hierzu Schwarz-Friesel 2014), was ein hohes Emotionspotenzial zur Folge hat und wodurch das Bedrohungspotenzial des ausbrechenden Krieges intensiviert wird:

19 Der metaphorische Ausdruck „Pulverfass Balkan“ steht seit seiner Entstehung dafür, die politischen, gesellschaftlichen und wirtschaftlichen Zustände auf dem Balkan aus einer westlichen Perspektive zu bezeichnen. Diese Metapher fungiert als ein vorurteilbeladenes Fremdbild, in dem sich die Selbstbilder bzw. die Projektionen und Zuschreibungen des Westens manifestieren und drückt die Sorgen des Westens, die eigenen Ängste, Erwartungen. Die Bilder des Westens beziehen sich z.B. auf die westliche Stabilität, Ordnung, Zivilisation, Zuverlässigkeit, Demokratie. Die Metapher übernimmt die Funktion eines Stereotyps, um dadurch das positive Selbstbild vom negativen Fremdbild zu konstruieren.

20 Paris (2002) verdeutlicht in seinem Beitrag „Kosovo and the Metaphor War", dass während der Kosovo-Krise (1989-1999) vier historische Kriegsmetaphern von der US-Verwaltung verwendet wurden, um die militärische Intervention zu unterstützen oder auch nicht: „das (andere) Vietnam“ , „,der (andere) Holocaust”, "die Münchner Konferenz" (S. 424) und schließlich die Metapher „Balkan Pulverfass" (,'Balkan powderkeg' (recalling the region's endemic instability and, specifically, the role that Balkan conflicts played in igniting World War I)" (S. 425).

21 Hydra, die (griech. Myth.): einer Schlange ähnliches Ungeheuer mit neun Köpfen (DUW 2015). 
(1) Ein jäher grausamer Schicksalsschlag hat [...] Das schreckliche Ereignis hat alle Herzen im Innersten aufgewühlt. Eine verruchte Hand hat das kostbare Leben des kaiserlichen Prinzen zerstört, der [...] Tief gebeugt, ins Innerste getroffen, empfanden die Völker der Monarchie tiefe grausame Fügung. Ihren Schmerz teilt die ganze Welt, die einig ist in dem Abscheu vor dem unmenschlichen Verbrechen, [...] In tiefer ernster Stunde erhebt sich der umflorte Blick zu unserem allergnädigsten Herrn, [...] (ANNO: Wiener Zeitung, 29.06.1914)

(2) Das Blut zweier braver und großer Menschen wurde vergossen. Die Hydra hochverräterischer Organisation, bisher versteckt, zischte auf und zeigte ihre sieben furchtbaren Rachen, und nunmehr ist der Krieg da. (ANNO: Wiener Zeitung, 29.07.1914)

Wenn wir einen Blick auf den Diskurs der ersten Berichterstattungstexte nach dem Attentat auf den Thronfolger und auf die offiziellen Stellungnahmen werfen, wird deutlich, dass die Standardreaktion auf dieses Ereignis neben einer Zorn- und Wutmotivik, wie in (3) „bis an den Hals satt haben", auch eine religiöse Motivik enthält, wie in (4) „ein heiliger Krieg, Blut- und Feuertaufe". Ausgehend von den Hauptbetroffenen - z.B. der Monarchie - findet in (3), (4) und (5) eine starke Gut-Böse-Polarisierung statt, die dem religiösen Bereich entstammt, wobei über das Böse mit „fester Hand bzw. mit der wackeren Armee", 'gesiegt' werden soll:

(3) Die gesamte Monarchie hat es bis an den Hals satt, von einem kleinen überreizten Volk unausgesetzt unterminiert, bedroht und wirtschaftlich auf das Schwerste geschädigt zu werden. Serbien ist heute noch die brennendste Lunte am europäischen Pulverfass. Soll eine Explosion verhindert werden, so muss der Brand rasch und mit fester Hand gelöscht werden. (ANNO: Neuigkeits Welt Blatt, 23.07.1914)

(4) Wenn der Funke ins Pulverfass fliegt, dann droht der Knall weithin, durch die Lunte alles zerschmetternd alles niederreißend. Wenn der Thronfolgermord durch Slawen für die Slawen ein solcher Funke ist, dann ist der Kampf der Staaten in der Mitte Europas, den dieser Funke hervorruft, ein gerechter Krieg, ein heiliger Krieg, ein Feuerkrieg, bei dem der Sieg unser sein muss. Aus dieser Blut- und Feuertaufe wird das beleidigte und das beschimpfte Ansehen unserer Monarchie, trotz des ihr zugedachten Verfalles, aufgerichtet und verjüngt hervorgehen. (ANNO: Allgemeiner Tiroler Anzeiger, 26.07.1914)

(5) Er wird lokalisiert bleiben, trotz des europäischen Gesamtkrieges. Serbien war allerdings der Funke, der das Pulverfass in die Luft gesprengt hat. Jetzt handelt es 
sich darum, den großen Weltbrand zu ersticken, der zum Himmel emporlodert. Der Funke Serbiens wird von unserer wackeren Armee leicht zertreten werden. (ANNO: Neues Wiener Journal, 03.08.1914)

(6) Serbien hat die Lunte an das europäische Pulverfass gelegt, Serbien war das Stemmeisen, mit dessen Hilfe der Dreiverband seinen verbrecherischen Einbruch unternahm. (ANNO: Vorarlberger Volksblatt, 29. 10. 1915)

Unternimmt man den Versuch, eine referenzielle Elaboration der Metapher Pulverfass Balkan durchzuführen, so können wir festhalten, dass die Instabilität nicht als ein lokaler Konflikt konzeptualisiert wird, sondern dass davon ganz Europa betroffen ist. Dadurch intensiviert sich das Gefühl des Bedrohungspotenzials. In (3)-(6) gehen die Textproduzenten davon aus, dass es sich bei diesem europäischen Krieg um einen von Serbien bzw. durch den Thronfolgermord verursachten Krieg handelt. Das Pulverfass stellt also einen weiteren Input für die Konzeptualisierung von "Serbien als Kriegsauslöser" bzw. "Thronfolgermord als Kriegsauslöser", der mit "Serbien (brennendste) Lunte" (3), "Serbien als der Funke" (4), "Thronfolgermord als der Funke" (5), "Serbien als der Stemmeisen" (6) versprachlicht wird.

Wie angedeutet erlebt die Metapher „Pulverfass Balkan“ mit den Konflikt- und Kriegsausbrüchen in den 1990er im ehemaligen Jugoslawien eine Neuauflage im öffentlichen Diskurs. Die Jahre nach 1990 waren die blutigsten Jahre im ehemaligen Jugoslawien. Die Kriege in Slowenien, Kroatien, Bosnien und Herzegowina und zuletzt auch im Kosovo, in Südserbien und Mazedonien haben während dieses Jahrzehntes nicht nur das ehemalige Jugoslawien, sondern den ganzen Balkan zum „explodierten Pulverfass" werden lassen. Der Untergang des Vielvölkerstaates, die Unabhängigkeitserklärungen der einzelnen Staaten, der wachsende Nationalismus wurden im Mediendiskurs sehr oft mit den metaphorischen Einheiten "Funke" oder "Lunte des Pulverfasses Balkan" versprachlicht. Aus den Arbeiten, die Metaphern aus dem Herkunftsbereich 'Krieg' untersuchen, resultiert, dass die Kriegsmetaphern ein hohes Emotionspotenzial besitzen. Durch die Kriegsmetapher "Pulverfass Balkan“ können von Seiten der Rezipienten mühelos Bedrohungs-, Gewalt- und Kriegsszenarien inferiert werden. Diese Szenarien bringen das Risikobewusstsein zum Ausdruck, wodurch die Sicherheit und Stabilität der modernen Welt beeinträchtigt wird. In (7)-(9) kann das mittels dieser Metapher aktivierte Gefahren- und Bedrohungspotenzial beim Rezipienten die Emotionen Furcht und Sorge auslösen, was die politischen und militärischen Handlungsoptionen als Lösungsmaßnahmen legitimiert. Außerdem wird in diesen 
Belegen eine Parallele zwischen den Kriegen in Bosnien und Herzegowina und im Kosovo gezogen und die übereinstimmenden Aspekte erörtert: „, militärischer Druck funktionierte in Bosnien, die Opfer der Milosevic-Kriege, mit immer neuen Toten":

(7) „Politischer, wirtschaftlicher und militärischer Druck haben in Bosnien funktioniert. Es gibt keinen Grund, warum ein ähnlicher Ansatz im Kosovo nicht eine ähnliche Wirkung haben sollte", sagte Solana. Europa werde keinen dauerhaften Frieden finden, wenn der Balkan ein Pulverfass bleibe. (St. Galler Tagblatt, 23.06.1998)

(8) SPIEGEL: Woher nehmen Sie eigentlich den Mut, in den Schluchten des Balkans gewaltsam mit Hilfe der Nato für Ordnung zu sorgen, einem seit Jahrhunderten unbeherrschbaren Pulverfass, wo sich schon Hitler und Stalin verrannt haben?

Fischer: [...] Auch der SPIEGEL wird es nicht aushalten, daß über die 300000 Opfer der Milosevic-Kriege hinaus immer weitere in Massengräbern oder sonst wo verscharrt werden - daß der ganze Balkan in Brand gesetzt wird. (Der Spiegel, 16/1999)

(9) Das Dilemma der Atlantischen Allianz: Sie hat trotz allen Säbelrasselns keine abgestimmte Strategie für die Eindämmung der ethnischen Konflikte in Europas Pulverfass. „Wir dürfen nicht in ein zweites Bosnien mit immer neuen Toten hineinschlittern", warnten die Amerikaner bei der Krisensitzung des Nato-Rats. Bundeskanzler Gerhard Schröder, der befürchtet, seine EU-Präsidentschaft könne von fortwährenden Kosovo-Gräueln überschattet werden, gab intern die Order aus, "die politische wie die militärische Option offenzuhalten und über den Zeitpunkt eines Eingreifens nachzudenken“ (Der Spiegel, 4/1999).

Obwohl der Krieg auf das ehemalige Jugoslawien lokalisiert war, wird in (10) mittels der Verwendung des Verbs "drohen“ das Bedrohungspotenzial eines neuen Krieges im gesamten Balkanraum impliziert, nämlich der Ausdehnung des Krieges auch auf Bulgarien und Griechenland. In (10) (auch in (11)) wird die Projektion des Herkunftsbereichs „Pulverfass" auf den Zielbereich „Kosovo" herausgelöst. Hier kann mittels der Verwendung des Lexems "Sprengstoff“ von Seiten der Rezipienten leicht das Szenario der Gefahr im Kosovo inferiert werden. Der Staatsoberhaupt Milosevic wird metaphorisch als „Brandstifter" bezeichnet, indem er als „Kriegshetzer" konzeptualisiert wird:

(10) Es ist der Präsident selbst, der Jugoslawien immer weiter zerstört. Deshalb ist das Kosovo keine innere Angelegenheit mehr, sondern ein Pulverfaß für den ganzen Balkan. Seine Explosion droht die Nachbarrepublik Makedonien zu zerreißen und 
damit Bulgarien und den Nato-Partner Griechenland in den Krieg gegeneinander zu stürzen. Dieser Sprengstoff muß dem Brandstifter jetzt aus der Hand genommen werden. (Die Zeit, 10.06.1998)

(11) Serbischer Terror im Pulverfass Kosovo

Die Albaner könnten die Demütigungen nicht länger ertragen, warnt Rexhep Ismajli vom Demokratischen Bund Kosovos: „Es genügt ein Funke, um das Pulverfass in die Luft zu jagen." (Der Spiegel, 21/1992)

In (11) erfolgt die Darstellung der politischen Lage im Kosovo aus der Perspektive vom selbst Betroffenen, einem lokalen Politiker. Mit Schwarz-Friesel (2014, S. 54) können wir festhalten, dass bei dieser Darstellung des Sachverhalts im Kosovo, die Strategie „Ereignisdarstellung durch einen Betroffenen“ mit „Bericht durch politische Autorität" kombiniert wird. Insgesamt entsteht ein mentales Szenario, das an die Emotion der Furcht gekoppelt ist. Der Textproduzent vermittelt durch den Wechsel von direkter und indirekter Rede die Sichtweise und die Wertung der politischen Lage durch den Betroffenen ohne weitere Bemerkungen.

In der Kriegsberichterstattung werden unter anderem auch religiöse Metaphern zum Einsatz gebracht, um etwas Unausweichliches oder Schicksalhaftes darzustellen. Durch das Lexem „Hölle" in (12) wird ein „Ort, Zustand großer Qualen; etwas Schreckliches, Furchteinflößendes, Unerträgliches“ (www.duden.de) beschrieben, das den Eindruck des Irrealen noch verstärkt (vgl. auch Schwarz-Friesel, 2014). Kirchhoff (2010, S. 186) zufolge verleihen religiöse Metaphern, wie hier "Hölle", "dem Krieg eine endzeitlicheschatologische Dimension“ und „weisen [...] Akteuren Rollen zu und beinhalten zudem eine eindeutige, starke moralische Bewertung" (Kirchhoff, 2010, S. 187). Im gleichen Beleg wird der ehemalige serbische Präsident durch die metaphorische Äußerung Wendekommunist bezeichnet, ein Neologismus mit negativer Konnotation. Der Textproduzent bringt mit der Wortgruppe legte der Wendekommunist die Lunten zu dem Pulverfass auf dem Balkan den Bereich des schwelenden Konflikts und des Staatsapparats in einen Zusammenhang, wobei Präsident Milosevic, mitsamt seinem Staatsapparat (Regierung, Militär), als Kriegsverursacher konzeptualisiert wird:

(12) Ganz Bosnien ist die Hölle - Ein Nürnberger Tribunal für Serben-Präsident Milosevic? Mit seiner nationalistischen Hetze legte der Wendekommunist die Lunten zu dem Pulverfass auf dem Balkan - und zündete sie selbst an. (Der Spiegel, 33/1992) 


\subsection{Analyse der Pressebelege konfliktarmer Zeiten: kommunistisches Jugoslawien und die neuen Balkanstaaten}

In diesem Teil werden Belege aus zwei Zeiträumen untersucht, die von Historikern als konfliktarme Zeiten bezeichnet werden: Der Erste Teil untersucht die Darstellung des kommunistischen Jugoslawiens in der deutschsprachigen Berichterstattung (1945-1989), der zweite Teil die Darstellung der Nachfolgestaaten des ehemaligen Jugoslawien.

Die Analyse hat ergeben, dass auch in diesen sogenannten konfliktarmen Zeiten der geografische Raum Balkan weiterhin als „Pulverfass" bewertet wird. Obwohl der Eindruck einer ruhigen Region in Jugoslawien herrschte, wurden dennoch im deutschsprachigen Mediendiskurs Belege eines möglichen Konfliktausbruches immer wieder gefunden. Aus dem Kontext der analysierten Belege mit der Metapher „Pulverfass Balkan“ im Zeitraum des kommunistischen Jugoslawiens konnten die Konzeptualisierungen „Konfliktauslöser als Vielvölkerstaat / Kommunismus / Fragmentierung / Kalter Krieg“ erzeugt werden, wie in den folgenden Belegen (13)-(16):

(13) Marschall Tito reitet ungebeugt auf dem Pulverfass Jugoslawien!

Beamte des westalliierten Spionage-Abwehrdienstes erhielten die Nachricht, dass die Sowjetunion versuchen werde, Tito innerhalb von sechs Monaten zu liquidieren. (Der Spiegel, 36/1949)

(14) Über eine Million Mazedonier wohnen heute in Jugoslawien, 180000 in Bulgarien, 40000 in Griechenland. Der Wunsch des dreigeteilten Volkes nach Wiedervereinigung ist die Lunte am Pulverfass Balkan: Die Bulgaren betrachten die Mazedonier als Bulgaren und möchten sie in ein Großbulgarisches Reich heimholen. [...] (Der Spiegel, 12/1967)

(15) Vor allem aber wäre es das Ende des Tito-Experiments, alle Südslawen in dem mehrsprachigen Vielvölkerstaat „Jugoslawien“ - bestehend aus sechs Bundesländern und zwei autonomen Gebieten - zu vereinen. Jugoslawien würde schon zu Titos Lebzeiten auseinanderbrechen - was erst für die Zeit nach Tito befürchtet worden war. Das Land wäre dann Streitobjekt der Großmächte, der Balkan wieder das Pulverfass Europas. (Der Spiegel, 52/1971)

(16) Jetzt stellen kommunistische Staaten immer häufiger Ansprüche auf Gebiete kommunistischer Nachbarn. Wird der Balkan wieder zum Pulverfass Europas? (Der Spiegel, 35/1978)

(17) Vor allem auf dem Balkan, dem ethnischen und religiösen Whirlpool zwischen 
Orient und Okzident: jahrhundertelang ein Jo-Jo von Fremdherrschern, Pufferzone und Schädelstätte rivalisierender Großmächte, schneller Brüter nationalrevolutionärer Ideen, schließlich Lunte und Pulverfass für einen Weltkrieg - Pavic hatte den Balkan im Auge. (Der Spiegel, 26/1988)

In (17) wird das jahrhundertelang herrschende negative Balkan-Bild hervorgehoben, indem der Balkan aufgrund ethnischer und religiöser Durchmischung metaphorisch mit einem „Whirlpool“, in dem es ständig brodelt, verglichen wurde. Der Textproduzent stellt außerdem den Balkan als Kreuzung zwischen West und Ost, in der unterschiedliche Kulturen, Traditionen und Zivilisationen aufeinandertreffen.

Mit der Millennium-Wende, also nach dem Zerfall des ehemaligen Jugoslawiens und der Gründung der Nachfolgestaaten, sehnte man sich nach einer friedlichen Entwicklung. Doch bleibt im deutschsprachigen Pressediskurs die metaphorische Äußerung „Pulverfass" weiterhin als Bewertungsmuster für den Balkan bestehen. Dadurch wird die Konzeptualisierung des ständigen Konfliktausbruchrisikos in diesem Raum erzeugt. Aus dem Kontext der Belege des zusammengestellten Korpus für diesen Zeitraum ergibt sich, dass als mögliche Faktoren für die Bewertung des Balkans als „Pulverfass" weiterhin der "Nationalismus" und die "ethnische Durchmischung" bleiben. Auffallend für diesen Zeitraum ist, dass „fundamentalistischer Islamismus, Korruption, Armut und Arbeitslosigkeit" als weitere Grundlagen für dieses Bewertungsmuster fungieren:

(18) Ohne Serbien ist die EU ein Torso, Serbien ein Pulverfass mit glimmender Lunte mitten in Europa. [...] Österreich ist seit eh und je kroatenfreundlich, viele Kroaten leben als österreichische Staatsbürger bei uns. Wir dürfen uns aber nicht auf die eingealterten Konflikte zwischen Kroaten und Serben einlassen, das ist ihre Sache. Fast 200.000 Serben leben ebenso geachtet und integriert bei uns. Nach ihren Stimmen schielt der nationalistische Strache. Ist er wirklich so kurzsichtig, ein Pulverfass Serbien einem Unionsmitglied Serbien vorzuziehen? Europa könnte auch hier die große Aussöhnung bringen! (Die Presse, 17.05.2008)

In (19) implizieren das konstituierte Risikoszenario des Auseinanderbrechens von Bosnien-Herzegowina sowie Lexeme wie "Mahnruf", "mahnen“, "warnen", "fürchten", eine negative Bewertung. Außerdem wird in (19) und auch in (20) das Bedrohungspotenzial durch die Lexeme „Fundamentalismus" und „Islamismus" bzw. den Vergleich „ein neues Palästina" erhöht: 
(19) Der Mahnruf des kroatischen Präsidenten klingt alarmierend: Ein Auseinanderbrechen der Republik Bosnien-Herzegowina - forciert vom dortigen Serbenführer Milorad Dodik - könne in einer Katastrophe enden, sagt Stipe Mesic gegenüber SPIEGEL ONLINE in Zagreb. Er warnt die Europäer vor der Gefahr eines neuen, blutigen Konflikts auf dem Balkan. Schlimmstenfalls werde ein fundamentalistischer Rumpfstaat der Muslime um Sarajevo übrigbleiben, „Europas Palästina und ein Hort für Terroristen“.[...] Solch ein Szenario ist für Kroatiens Staatschef eine Horrorvision. Nach dem Auseinanderbrechen von BosnienHerzegowina "hätten wir als Rest dort einen islamischen Staat, ein neues Palästina. Und dieser Staat wäre umzingelt von Feinden". Um überhaupt existieren zu können, wäre dieses Gebilde auf die Unterstützung durch fundamentalistische Regime angewiesen und auf die Zusammenarbeit mit radikalen Islamisten. "In Sarajevo würde dann ein Zentrum des Terrorismus entstehen“. Europa nehme Dodik auf die leichte Schulter, fürchtet Mesic, "die Serbische Republik ist ein Pulverfass, das jederzeit in die Luft fliegen kann“. (Der Spiegel, 08.10.2008)

(20) Ein weiterer Beitrag in dem Heft widmet sich der Westbalkanregion als Rekrutierungsfeld für militante Islamistengruppen. Fast ein Viertel der aus Europa stammenden ausländischen Kämpfer des Islamischen Staates stammen aus Albanien, Bosnien und Herzegowina, dem Kosovo und Mazedonien. Da der IS im Irak und in Syrien gerade schwer unter Druck steht, könnten zurückkehrende ISKämpfer in dem explosiven ethnischen und religiösen Gemisch am Westbalkan noch wie der Funken in einem Pulverfass wirken. (Die Presse, 09.07.2017)

Ähnlich auch in (21)-(22) inferieren die Lexeme "riskieren“, ,teuer werden“, „alles aufs Spiel setzen“, „Funke“, „,Pulverfass", „Warnung ${ }^{\prime}$, ,Feuer ${ }^{\prime \prime}$, ,Nationalismus" das Risikoszenario:

(21) „In diesem Pulverfass reicht ein Funke“

Armeechef André Blattmann will die Präsenz der Swisscoy im Kosovo verlängern. Wenn die Truppen zu früh zurückgezogen würden, riskiere man alles bisher Erreichte. Das könnte für die Schweiz teuer werden. [...] Was im Kosovo passiere, habe einen direkten Einfluss auf die Schweiz. Wenn sich die Truppen zu früh zurückzögen, setze man alles aufs Spiel, was bisher erreicht worden sei. „Ohne Truppenpräsenz reicht ein Funke in diesem Pulverfass, und wir würden um Jahre zurückgeworfen." Nur schon rein finanziell käme das viel teurer zu stehen. (Tages-Anzeiger, 27.12.2012)

(22) [...] Die Warnung der Europäischen Union und der Dayton-Garantiestaaten an Bosniens Politiker ist eindeutig: Hört auf mit der Selbstbereicherung! Und: Spielt 
nicht mit dem Feuer des Nationalismus! Der Balkan ist ein Pulverfass. Immer noch. (Euronews, 28.02.2014)

Auch in den jüngeren Pressebelegen gilt die Region weiterhin als instabil. In (23)- (24) stützt das Konzept der manifesten "Armut", "Korruption" oder "Nationalismus" die Bewertung dieser Staaten als „Pulverfass vom Balkan“:

(23) Und auch die linksnationalistische Partei "Vetëvendosje“ (,Selbstbestimmung“), die den Kampf gegen Korruption und Vetternwirtschaft propagiert, liegt nur noch knapp dahinter. Womöglich entscheiden am Ende ein paar Tausend Stimmen darüber, ob der Westbalkan wieder zum Pulverfass wird. (Der Spiegel, 11.06.2017)

(24) Es brodelt im Kosovo. Wird der von Armut, Korruption und dem Nationalitätenkonflikt geprägte Kleinstaat mit weniger als zwei Millionen Einwohnern erneut zum Pulverfass des Balkans? (Der Spiegel, 17.04.2017)

Der Beleg (25) zeigt, dass sich die Denkweise der Balkan-Politiker kaum verändert hat. Auch nach fast einem Jahrhundert gilt die Feststellung von Keyslinger (1931, S. 339) „Der Geist des Balkans als solcher ist der Geist ewigen Streits. Bewohnen ihn primitive Rassen, so bietet er das Urbild des Urkampfes gegen alle."

(25) Pulverfass Balkan. Region im Schwebezustand

Regionales Denken und Handeln ist Politikern auch 25 Jahre nach dem Untergang des alten Jugoslawiens ziemlich fremd. Anstatt als Gemeinschaft gestärkt gegenüber EU und Nato aufzutreten, sucht jede der ehemaligen Teilrepubliken ihren eigenen Weg nach Europa. Die bilateralen Beziehungen sind zwar vielfältig, werden aber oft noch von Feindschaft und Misstrauen aus der Kriegszeit belastet.

(St.Galler Tagesblatt, 04.06.2017)

\section{Fazit}

Im Folgenden werden wir versuchen anhand des Netzwerkmodells der Blendingtheorie die Ergebnisse der Analyse bezüglich der Bedeutung dieser Metapher in den Medienberichten aus verschiedenen Zeiträumen und Bereichen auf ihre Unterschiede und Gemeinsamkeiten hin zu überprüfen. 
Aus den analysierten Belegen der Zeit der akuten Krisen (Erster Weltkrieg und postjugoslawische Kriege) erzeugen sich die Metapher "Serbien als europäisches Pulverfass" und "Westbalkan als Pulverfass Europas". Nach der Blendingtheorie können wir diese Metapher wie folgt darstellen:

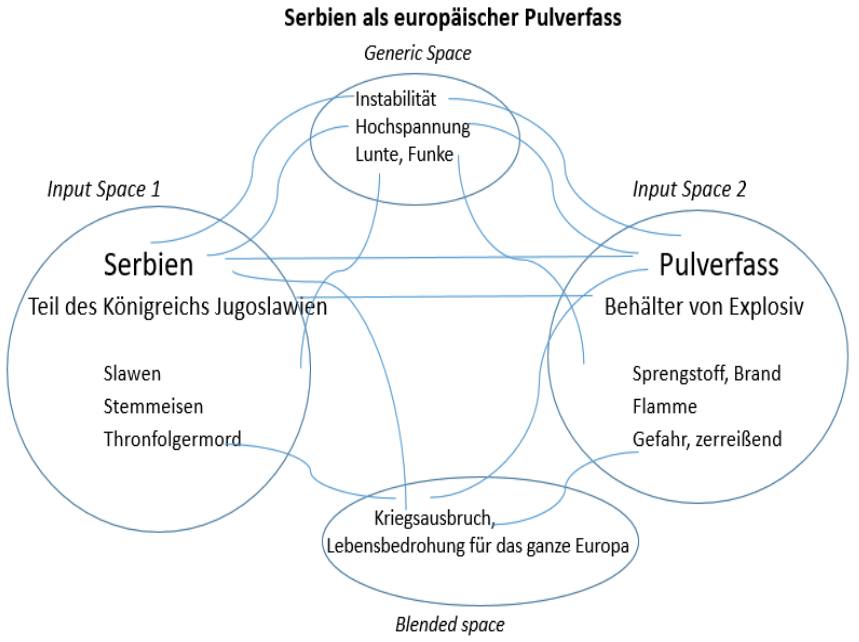

Fig 2. Blend von "Serbien als europäischer Pulverfass"

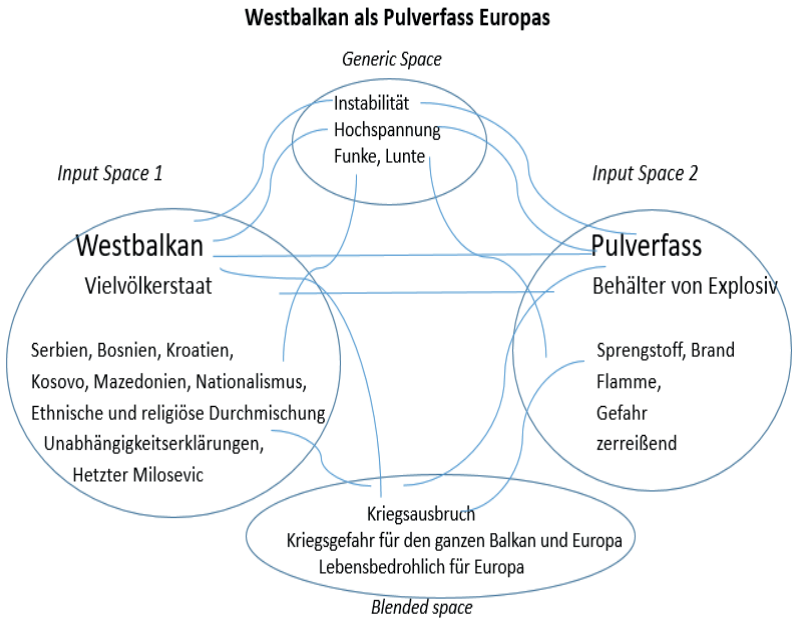

Fig 3. Blend von „Westbalkan als Pulverfass Europas"

Auch in der konfliktarmen Zeit des kommunistischen Jugoslawiens wird Jugoslawien weiterhin mit der Metapher "Pulverfass" bewertet. Aus den Belegen von diesem 
Zeitraum erzeugt sich die Metapher „Jugoslawien als Pulverfass Europas”. Nach der Blendingtheorie können wir diese Metapher wie folgt analysieren. Mit dieser Metapher wird der „blended space Kriegsgefahr für Europa”, "Gefährdung der europäischen westlichen Werte" und "Lebensbedrohlich für Europa" ausgelöst, der in den beiden Inputs nicht vorkommt.

\section{Jugoslawien als Pulverfass Europas}

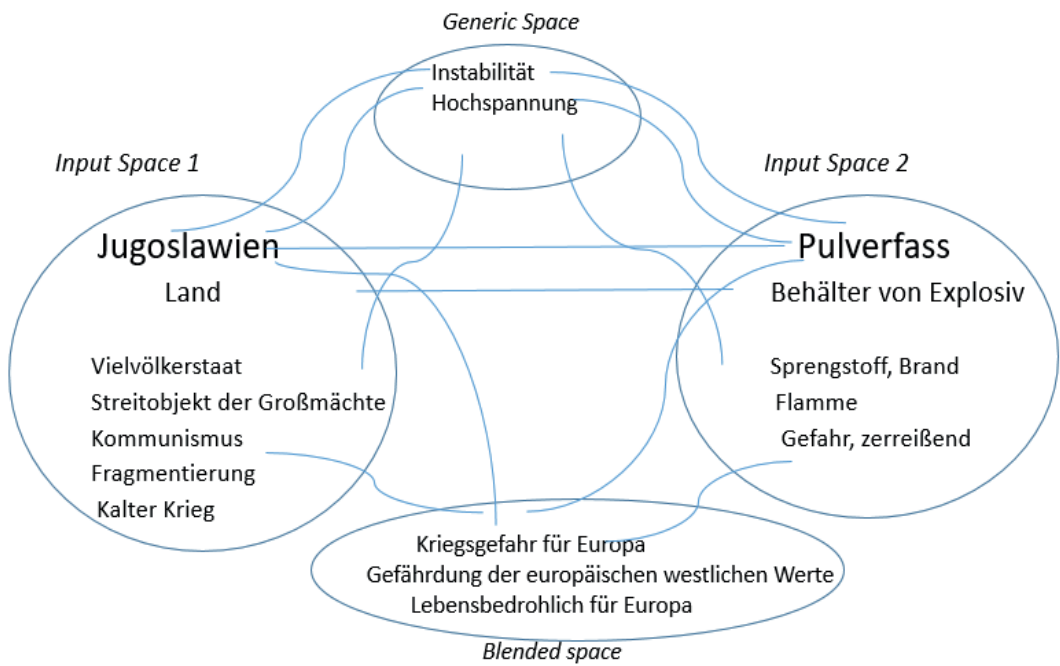

Fig 4. Blend von „Jugoslawien als Pulverfass Europas"

In dem Zeitraum nach der Millenniumswende, also nach dem Kriegsende in allen Ländern Ex-Jugoslawiens bis zur Gegenwart, wird der Westbalkan wegen der politischen Ereignisse, wirtschaftlichen Lage aber auch wegen ethnischer und religiöser Durchmischung bzw. vor allem wegen der Gefahr des islamischen Fundamentalismus weiterhin als „Pulverfass" referenzialisiert. Aus den analysierten Belegen erzeugt sich die Metapher „Kosovo als Pulverfass Balkans“ bzw. „Bosnien als Pulverfass Balkans“. Nach der Blendingtheorie können wir diese Metapher wie folgt darstellen: 


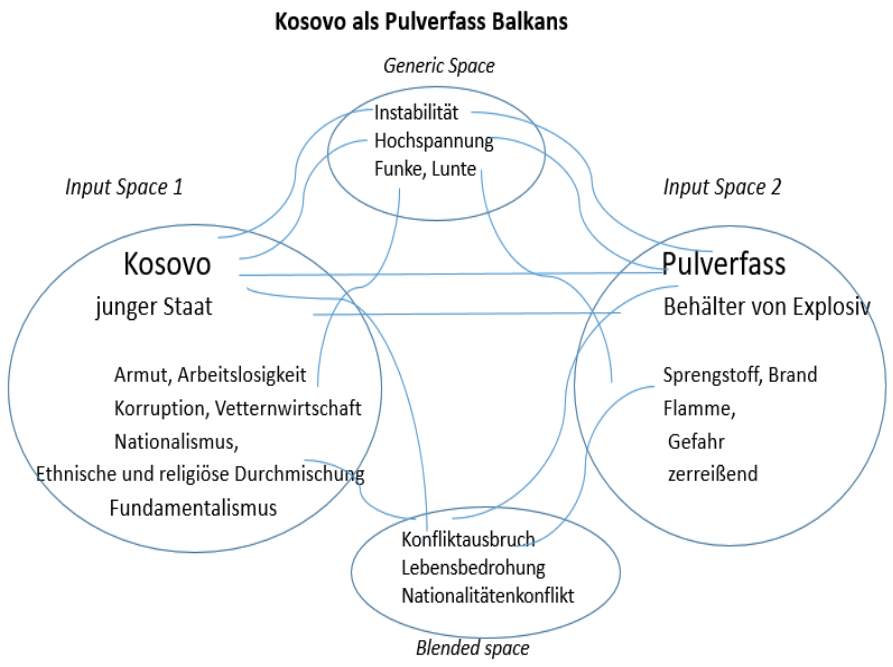

Fig 5. Blend von „Kosovo als Pulverfass Balkans"

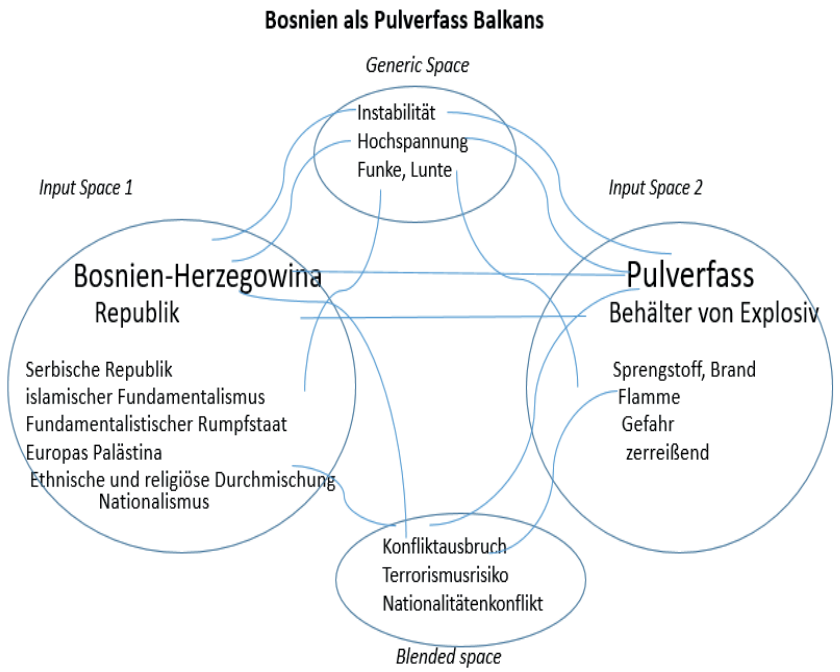

Fig 6. Blend von „Bosnien als Pulverfass Balkans"

Wie man aus dieser Analyse entnehmen kann, hat sich die Bedeutung dieser Metapher in den Medienberichten in diesen hier untersuchten Zeiträumen nicht viel verändert. Gemeinsamkeiten bezüglich der konzeptuellen Merkmale, die die metaphorische Äußerung Pulverfass Balkan in allen Zeiträumen inferiert, sind: „ethnische und religöse Durchmischung“, "Nationalismus", "Instabilität", „Primitivität“. Kleine Unterschiede kann man in den konfliktarmen Zeiträumen merken, wo noch folgende Merkmale in den 
analysierten Belegen vorkommen: „Kommunismus" und "Kalter Krieg" (in der Jugoslawischen Zeit), „Korruption“, "fundamentalistischer Islamismus und Gefahr vom Terrorismus", der nicht nur Balkan, sondern die ganze Welt bedroht (in der neueren Zeit).

\section{Abschließende Bemerkungen}

Das grundsätzliche Ziel dieses Beitrags war es, eine systematische Beschreibung der Metapher „Pulverfass Balkan“ im deutschen Mediendiskurs durchzuführen. Die Analyse hat sich auf das ehemalige Jugoslawien beschränkt. Aus methodologischen Gründen wurden die Presseberichte mit der Metapher „Pulverfass Balkan“ in zwei Teilen untersucht: Zum einen sind Presseberichte über akute Krisen (Erster Weltkrieg und postjugoslawische Kriege) analysiert worden, zum anderen solche über konfliktarme Zeiten (kommunistisches Jugoslawien und die neuen Balkanstaaten). Diese Metapher datiert schon von der Zeit vor dem Ersten Weltkrieg. Sicherlich ist sie mit den Kriegsausbrüchen im westlichen Vokabular verstärkt im Bewusstsein verankert worden und wurde dadurch zum allgemeinen Bewertungsmuster der Balkanwahrnehmung. Die Analyse hat ergeben, dass die Metapher „Pulverfass Balkan“ folgende konzeptuelle Merkmale inferiert: „Instabilität“, „Primitivität", „Unzuverlässigkeit“, „ethnische und religiöse Durchmischung“, "Gewalt", "Korruption", „mangelnde Demokratie" und "fundamentalistischer Islamismus". Der Gebrauch der Metapher „Pulverfass Balkan“ im Mediendiskurs hat die Funktion Einstellungen und Bewertungen des Westens zu vermitteln sowie Meinungen über die Lage auf dem Balkan im Allgemeinen zu transportieren.

Zusammenfassend kann festgehalten werden, dass der Balkan seit mehr als einem Jahrhundert - wegen der politischen und historischen Ereignisse - als "Pulverfass" referenzialisiert wird. In den untersuchten Belegen wurde die metaphorische Äußerung „Pulverfass" immer für den ganzen Balkan verwendet, dessen Instabilität das ganze Europa bedroht. Hinter der Metapher "Pulverfass" verbergen sich "Konflikte", „Kriege“, "staatliche oder individuelle Verbrechen“, „Brutalitäten“ und „Aggressionen“.

Man kann zu Recht sagen, dass der Balkan und insbesondere das ehemalige Jugoslawien seit hundert Jahren von diesem schlechten Leumund verfolgt wird. Wegen der vielen ungelösten politischen Probleme wird diese Region im Politikdiskurs aber auch im Mediendiskurs weiterhin als „Pulverfass” bezeichnet. Mit Veichtlbauer (2001, S. 133) können wir schlussfolgern: „Der Balkan ist auf schicksalshafte Weise geblieben, was er immer war." Diese Untersuchung soll ein Ausblick auf die bevorstehende Forschung 
darstellen. Die Wahrnehmung von Balkan aus der Innenperspektive ist der kommenden Untersuchung vorbehalten.

Begutachtung: Extern begutachtet.

Interessenkonflikt: Es besteht kein Interessenkonflikt.

Finanzielle Förderung: Dieser Beitrag wurde von keiner Institution finanziell unterstützt.

Peer-review: Externally peer-reviewed.

Conflict of Interest: The authors hve no conflict of interest to declare.

Grant Support: The authors declared that this study has received no financial support.

\section{Literaturverzeichnis}

Becker, M. J. (2014). Gewaltmetaphern im französischen Präsidentschaftswahlkampf 2011/12. In M. SchwarzFriesel \& J.-H. Kromminga (Hrsg.), Metaphern der Gewalt, Konzeptualisierungen von Terrorismus in den Medien vor und nach 9/11, (S. 197-215). Tübingen: Francke.

Bjelić, D. (2002). Introduction: Blowing Up the “Bridge”. In D. I. Bjelić \& O. Savić (eds.), Balkan as Metaphor Between Globalization and Fragmentation, (1-22). Cambridge u.a.: The MIT Press.

Czachur, W. (2015). Semantischer und funktionaler Wandel von Metapher und Metonymie. Polnische Wirtschaft im deutschen Mediendiskurs zur Wirtschaftskrise. In C. Spieß \& K.-M. Köpcke (Hrsg.), Metapher und Metonymie Theoretische, methodische und empirische Zugänge (Empirische Linguistik/Empirical Linguistics Bd.1), (S. 227-246). Berlin, München, Boston: Walter de Gruyter.

Dudenredaktion (Hrsg.) (2013) [1992]. DUDEN. Band 11. Redewendungen. Wörterbuch der deutschen Idiomatik. 4. Auflage. Berlin: Dudenverlag.

Dudenredaktion (Hrsg.) (2015) [1983]. DUDEN Deutsches Universal Wörterbuch. Das umfassende Bedeutungswörterbuch der deutschen Gegenwartssprache. 8. Auflage. Berlin: Dudenverlag.

Fauconnier, G. \& Turner, M. (2002). The Way We Think: Conceptual Blending and the Mind's Hidden Complexities. New York: Basic Books.

Fauconnier, G. (1997). Mappings in Thought and Language. Cambridge: University Press.

Geulen, C. (2002).,Enemy Mine': Über unpolitische Feindschaft. In C. Geulen, A. v. d. Heiden \& B. Liebsch (Hrsg.) Vom Sinn der Feindschaft, (S. 77-108). Berlin Akademie Verlag.

Ghilarducci, M. (2017). Die Funktion der Tropen in Aleksandr Prochanovs literarischer Publizistik. Eine dekonstruktivistische Annäherung. In Zeitschrift für Slawistik 62 (1), 178-190.

Goldsworthy, V. (2002). Invention and In(ter)vention: The Rhetoric of Balkanization. In D. I. Bjelić \& O. Savić (eds.). Balkan as Metaphor Between Globalization and Fragmentation, S. 25-38. Cambridge u.a: The MIT Press.

Kaplan, R. I. (2000). The coming anarchy. Shattering the Dreams of the Post Cold War. Toronto: Random Haus. Keisinger, F. (2008). Unzivilisierte Kriege im zivilisierten Europa? Die Balkankriege und die öffentliche Meinung in Deutschland, England und Irland. 1876-1913. Paderborn: Ferdinand Schöningh. 
Keyserling, H. (1931). Das Spektrum Europas. Stuttgart \& Berlin: Deutsche Verlags-Anstalt.

Kirchhoff, S. (2010). Krieg mit Metaphern. Mediendiskurse über 9/11 und den „War on Terror". Bielefeld: transcript Verlag.

Koschmal, W. (2009). Wahlverwandtschaften: (Mittel-)Europa, die Metapher und der Essay. In Zeitschrift für Slawistik, 54(4), 445-456.

Kövecses, Z. (2010) [2002]. Metaphor: A Practical Introduction, 2nd edn. New York: Oxford University Press.

Lakoff, G. \& Johnson, M. (2003) [1980]. Metaphors We Live by. Chicago: The University of Chicago Press.

Lakoff, G. \& Johnson, M. (2008) [1997]. Leben in Metaphern. Konstruktion und Gebrauch von Sprachbildern, 6 Auflage. Heidelberg: Carl-Auer-Verlag.

Liebert, W.-A. (2014). Ultimatives Kunstwerk mit Todesfolge. Das 9 /11-Blending Karlheinz Stockhausens im Pressegespräch am 16.09.2001. In M. Schwarz-Friesel \& J.-H. Kromminga (Hrsg.), Metaphern der Gewalt, Konzeptualisierungen von Terrorismus in den Medien vor und nach 9/11, (S. 25-50). Tübingen: Francke.

Liebert, W.-A. (2015). Metaphern der Desillusionierung. Die Bereiche Theater, Höhle, Traum, Phantom, Gefängnis, Simulation und Hologramm als Ressource für Blendings. In C. Spieß \& K.-M.I Köpcke (Hrsg.), Metapher und Metonymie Theoretische, methodische und empirische Zugänge (Empirische Linguistik/Empirical Linguistics Bd.1), (S. 111-141). Berlin, München, Boston: Walter de Gruyter.

Mišev, R. (2001). Der Balkan - Pulverfass oder Schuld und Sühne der europäischen Diplomatie?. In P. Angelova \& J. Veichtlbauer (Hrsg.), Pulverfass Balkan. Mythos oder Realität: Internationales Symposium Rousse Oktober 1998 (Schriftenreihe der Elias Canetti Gesellschaft Band 3), (S. 223-232). St. Ingbert: Röhrig Universitätsverlag

Paris, R. (2002). Kosovo and the Metaphor War. Political Science Quarterly 117(3), 423-450.

Schmitt, R. (2017). Systematische Metaphernanalyse als Methode der qualitativen Sozialforschung. Wiesbaden: Springer.

Schröder, U. (2012). Kommunikationstheoretische Fragestellungen in der kognitiven Metaphernforschung. Eine Betrachtung von ihren Anfängen bis zur Gegenwart. Tübingen: Narr Francke Attempto.

Schwarz-Friesel, M. (2013) [2007]. Sprache und Emotion, 2. Aufl. Tübingen: Narr .

Schwarz-Friesel, M. (2014). „Hydra, Krake, Krebsgeschwür, Sumpf, Killer-GmbH, Franchise-Unternehmen und Nebenwolke". Perspektivierung und Evaluierung von islamistischen Terrorismus durch Metaphern im deutschen Pressediskurs nach 9/11. In M. Schwarz-Friesel \& J.-H. Kromminga (Hrsg.), Metaphern der Gewalt, Konzeptualisierungen von Terrorismus in den Medien vor und nach 9/11, (S. 51-74). Tübingen: Francke.

Schwarz-Friesel, M. (2015). Metaphern und ihr persuasives Inferenzpotenzial. Konzeptualisierungen des islamistischen Terrorismus nach 9/11 im massenmedialen Diskurs. In C. Spieß \& K.-M. Köpcke (Hrsg.), Metapher und Metonymie Theoretische, methodische und empirische Zugänge (Empirische Linguistik/ Empirical Linguistics Bd.1), (S. 143-160). Berlin, München, Boston: Walter de Gruyter.

Skirl, H. \& Schwarz-Friesel, M. (2013). Metapher. Heidelberg: Universitätsverlag Winter. 
Spieß, C. \& Köpcke, K.-M. (2015). Metonymie und Metapher - Theoretische, methodische und empirische Zugänge. Eine Einführung. In C. Spieß \& K.-M. Köpcke (Hrsg.), Metapher und Metonymie Theoretische, methodische und empirische Zugänge (Empirische Linguistik/Empirical Linguistics Bd.1), (S. 1-21). Berlin, München, Boston: Walter de Gruyter.

Sundhaussen, H. \& Clewing, K. (Hrsg.) (2016) [2004]. Lexikon zur Geschichte Südosteuropas. 2. Auflage. Wien u.a.: Böhlau.

Sundhaussen, H. (1999). Europa balcanica. Der Balkan als historischer Raum Europas. Geschichte und Gesellschaft: Zeitschrift für historische Sozialwissenschaften, 25(4), 626-653.

Todorova, M. (2009). Imagining the Balkans. Oxford: University Press.

Ulich, D. \& Mayring, P. (2003). Psychologie der Emotionen. Stuttgart: Kohlhammer.

Veichtlbauer, J. (2001). Das innere Ausland - der Balkan als Hinterhof Europas. In P. Angelova \& J. Veichtlbauer (Hrsg.), Pulverfass Balkan. Mythos oder Realität: Internationales Symposium Rousse Oktober 1998 (Schriftenreihe der Elias Canetti Gesellschaft Band 3), (S. 125-149). St. Ingbert: Röhrig Universitätsverlag.

Wierzbicka, A. (1999). Emotions Across Languages and Cultures: Diversity and Universals. Paris: Cambridge University Press.

Ziem, A. (2015). (Konzeptuelle) Metaphern in der Kognitiven Konstruktionsgrammatik. In C. Spieß \& K.-M. Köpcke (Hrsg.), Metapher und Metonymie Theoretische, methodische und empirische Zugänge (Empirische Linguistik/Empirical Linguistics Bd.1), (S. 51-80). Berlin, München, Boston: Walter de Gruyter. 
